\title{
Adrenal Ewing's Sarcoma in an Elderly Man
}

\author{
Kazuyoshi Toda ${ }^{1}$, Sumiyasu Ishii ${ }^{1}$, Hidetoshi Yasuoka ${ }^{1}$, Masaki Nishioka ${ }^{1}$, \\ Takayuki Kobayashi ${ }^{1}$, Kazuhiko Horiguchi ${ }^{1}$, Takuya Tomaru ${ }^{1}$, Atsushi Ozawa ${ }^{1}$, \\ Nobuyuki Shibusawa ${ }^{1}$, Tetsurou Satoh ${ }^{1}$, Hiromi Koshi ${ }^{2}$, Atsuki Segawa ${ }^{2}$, Shin-ichi Shimizu ${ }^{2}$, \\ Tetsunari Oyama ${ }^{2}$ and Masanobu Yamada ${ }^{1}$
}

\begin{abstract}
:
Ewing's sarcoma usually arises in the bones of children and adolescents. We herein report a 74-year-old man with Ewing's sarcoma in the adrenal gland. The diagnosis was confirmed by a genetic test, pathological studies, and several imaging studies. He already had multiple liver metastases when he was transferred to our hospital and died on the 37th day. The diagnosis was further confirmed by autopsy studies. Adrenal Ewing's sarcoma is very rare, and our patient was older than other reported cases. Ewing's sarcoma should be considered even in elderly patients with adrenal tumors.
\end{abstract}

Key words: adrenal gland, Ewing's sarcoma, elderly

(Intern Med 57: 551-555, 2018)

(DOI: 10.2169/internalmedicine.8892-17)

\section{Introduction}

Adrenal incidentalomas are defined as adrenal tumors identified by imaging studies without clinical suspicion of adrenal diseases. Adrenal incidentalomas have been detected in approximately $4 \%$ of patients that underwent highresolution imaging studies (1). A critical point in dealing with adrenal incidentaloma is determining whether or not the tumor is malignant. It is recommended that malignancy be considered when the tumor is larger than $4 \mathrm{~cm}(1,2)$.

Ewing's sarcoma is the second-most common bone tumor in children and adolescents and affects $250-400$ patients in the United States each year. The peak age of onset is 15 years, and $80 \%$ of cases are diagnosed before 18 years of age. Chemotherapy has greatly improved the survival rate for patients with localized tumors in recent years, but the prognosis remains poor in those with metastasis or recurrent tumors $(3,4)$.

We herein report our findings in treating a 74-year-old man with Ewing's sarcoma in the adrenal gland. The diagnosis was confirmed by a genetic test, pathological studies including autopsy, and several imaging studies. To our knowledge, our patient is the oldest case of adrenal Ewing's sarcoma.

\section{Case Report}

A 74-year-old man visited another hospital because of abdominal pain on his right side in July 2013. Computed tomography (CT) demonstrated a tumor on his right adrenal gland (Fig. 1A). The tumor was $6.7 \mathrm{~cm}$ in size and appeared heterogeneous, which is not consistent with benign adrenal incidentaloma. ${ }^{18}$ F-fluorodeoxyglucose-positron emission tomography (FDG-PET) revealed the enhanced uptake of the tracer in the tumor, suggesting that the tumor was malignant (Fig. 1B). The maximum standardized uptake value (SUVmax) was 7.6. No significant uptake was observed in other organs, suggesting that the adrenal tumor was not a metastatic lesion of malignant tumors in other organs. The tumor appeared to be hypointense on T1-weighed magnetic resonance imaging (MRI) (Fig. 1C). The T2-weighed image confirmed that the internal signal was heterogeneous (Fig. 1D). Metaiodobenzylguanidine (MIBG) scintigraphy findings were negative (Fig. 1E). In September, the patient underwent open surgery, but the pathological diagnosis was

${ }^{1}$ Department of Medicine and Molecular Science, Gunma University Graduate School of Medicine, Japan and ${ }^{2}$ Department of Diagnostic Pathology, Gunma University Graduate School of Medicine, Japan

Received: January 18, 2017; Accepted: August 3, 2017; Advance Publication by J-STAGE: December 21, 2017

Correspondence to Dr. Sumiyasu Ishii, sishii@gunma-u.ac.jp 

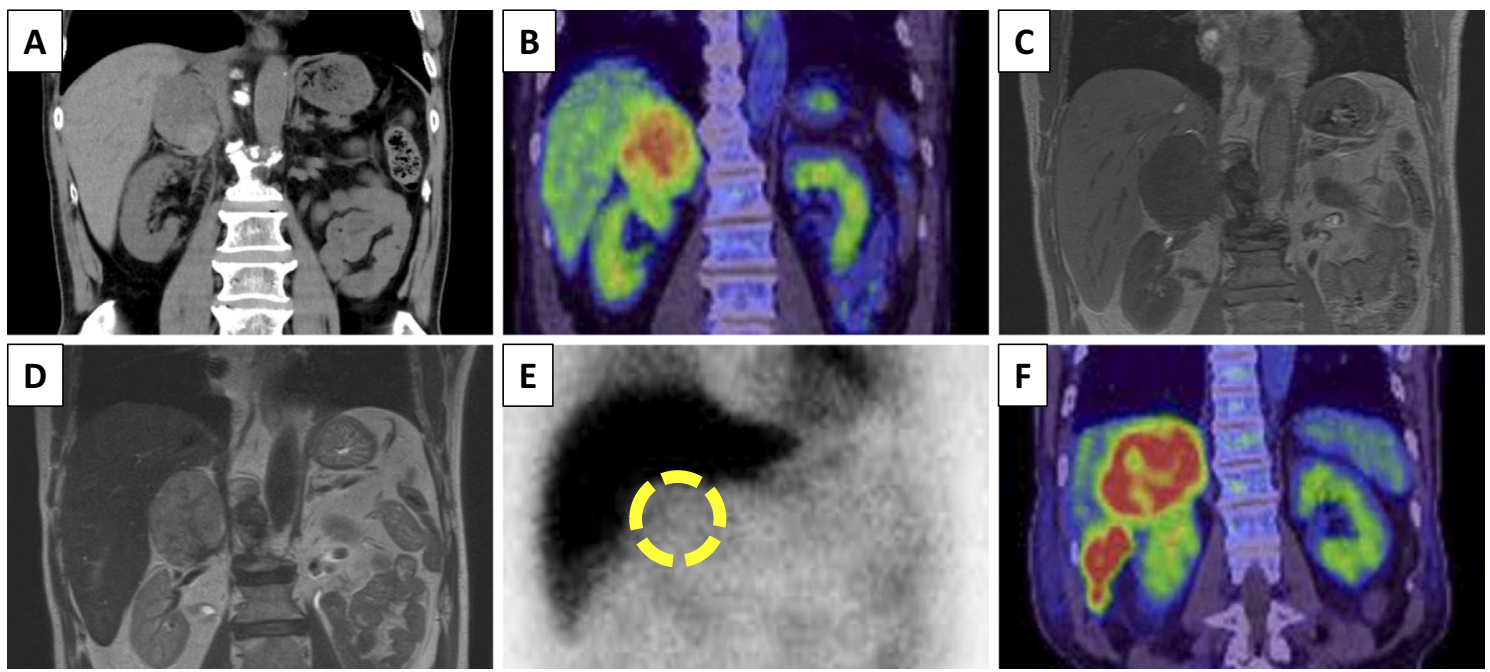

Figure 1. Imaging studies of the adrenal tumor before surgery (A-E) and recurrent liver tumors after surgery (F). A: Computed tomography studies demonstrated a large heterogeneous tumor in the right adrenal gland. B: An ${ }^{18} \mathrm{~F}$-fluorodeoxyglucose-positron emission tomography image of the adrenal tumor showed the enhanced uptake of the tracer in the tumor. $C$ : The tumor was hypointense on T1-weighted magnetic resonance imaging. D: T2-weighted magnetic resonance imaging showed that the internal signal was heterogeneous. E: Metaiodobenzylguanidine scintigraphy findings were negative. The position of the tumor is indicated by the yellow circle. $\mathrm{F}:{ }^{18} \mathrm{~F}$-fluorodeoxyglucose-positron emission tomography studies in December revealed multiple recurrence of the malignant tumor in the liver.

Table. Laboratory Findings on Admission.

\begin{tabular}{lclclc}
\hline Hematology & & Blood Chemistry & & Hormones \\
Hematocrit & $25.6 \%$ & Total protein & $6.2 \mathrm{~g} / \mathrm{dL}$ & Corticotropin & $73.9 \mathrm{pg} / \mathrm{mL}$ \\
Hemoglobin & $8.6 \mathrm{~g} / \mathrm{dL}$ & Albumin & $3.0 \mathrm{~g} / \mathrm{dL}$ & Cortisol & $24.9 \mu \mathrm{g} / \mathrm{dL}$ \\
Red blood cell & $295 \times 10^{4} / \mu \mathrm{L}$ & Aspartate aminotransferase & $37 \mathrm{IU} / \mathrm{L}$ & DHEA-S & $1,475 \mathrm{ng} / \mathrm{mL}$ \\
Platelet & $20.8 \times 10^{4} / \mu \mathrm{L}$ & Alanine aminotransferase & $30 \mathrm{IU} / \mathrm{L}$ & Urinary free cortisol & $19.3 \mu \mathrm{g} / \mathrm{day}$ \\
White blood cell & $6,000 / \mu \mathrm{L}$ & Lactate dehydrogenase & $471 \mathrm{IU} / \mathrm{L}$ & Plasma renin activity & $0.4 \mathrm{ng} / \mathrm{mL} / \mathrm{h}$ \\
$\quad$ Neutrophil & $66.5 \%$ & Alkaline phosphatase & $493 \mathrm{IU} / \mathrm{L}$ & Aldosterone & $58.2 \mathrm{pg} / \mathrm{mL}$ \\
$\quad$ Eosinophil & $2.3 \%$ & $\gamma$-glutamyl transpeptidase & $121 \mathrm{IU} / \mathrm{L}$ & Adrenaline & $0.02 \mathrm{ng} / \mathrm{mL}$ \\
$\quad$ Basophil & $0.5 \%$ & Blood urea nitrogen & $15 \mathrm{mg} / \mathrm{dL}$ & Noradrenaline & $0.50 \mathrm{ng} / \mathrm{mL}$ \\
$\quad$ Monocyte & $0.6 \%$ & Creatinine & $0.66 \mathrm{mg} / \mathrm{dL}$ & & $135.0 \mathrm{ng} / \mathrm{mL}$ \\
$\quad$ Lymphocyte & $20.7 \%$ & Sodium & $131 \mathrm{mEq} / \mathrm{L}$ & Tumor markers & $1,120.0 \mathrm{pg} / \mathrm{mL}$ \\
Coagulation & & $4.7 \mathrm{mEq} / \mathrm{L}$ & Neuro-specific enolase & \\
Fibrinogen & $380 \mathrm{mg} / \mathrm{dL}$ & Chloride & $98 \mathrm{mEq} / \mathrm{L}$ & ProGRP & \\
Prothrombin time & $86 \%$ & Glucose & $115 \mathrm{mg} / \mathrm{dL}$ & & \\
APTT & $34.1 \mathrm{sec}$ & Hemoglobin A1c & $7.6 \%$ & & \\
FDP & $22.7 \mu \mathrm{g} / \mathrm{mL}$ & Total cholesterol & $120 \mathrm{mg} / \mathrm{dL}$ & & \\
D-dimer & $8.7 \mu \mathrm{g} / \mathrm{mL}$ & C-reactive protein & $7.34 \mathrm{mg} / \mathrm{dL}$ & & \\
\hline APTT & & & & \\
\hline
\end{tabular}

APTT: activated partial thromboplastin time, FDP: fibrin/ fibrinogen degradation product, DHEA-S: dehydroepiandrosterone sulfate, ProGRP: pro-gastrin-releasing peptide

obscure. In November, he suffered from a slight fever and general fatigue. FDG-PET/CT studies in December revealed multiple recurrence of the malignant tumor in the liver (Fig. 1F). The patient was transferred to our hospital in January 2014.

His Eastern Cooperative Oncology Group (ECOG) performance status was 1 on admission. He did not present any abnormal findings on a physical examination, except for a slight fever. His blood pressure was well-controlled by can- desartan. He did not have any symptoms of Cushing's syndrome or phenochromocytoma. Blood tests demonstrated anemia, elevated levels of liver enzymes, C-reactive protein (CRP), neuro-specific enolase (NSE), and pro-gastrinreleasing peptide (ProGRP) (Table). His urinary free cortisol level was not elevated, although the levels of corticotropin and cortisol in the plasma were slightly high, probably due to stress. His aldosterone and catecholamine levels were within normal limits. 

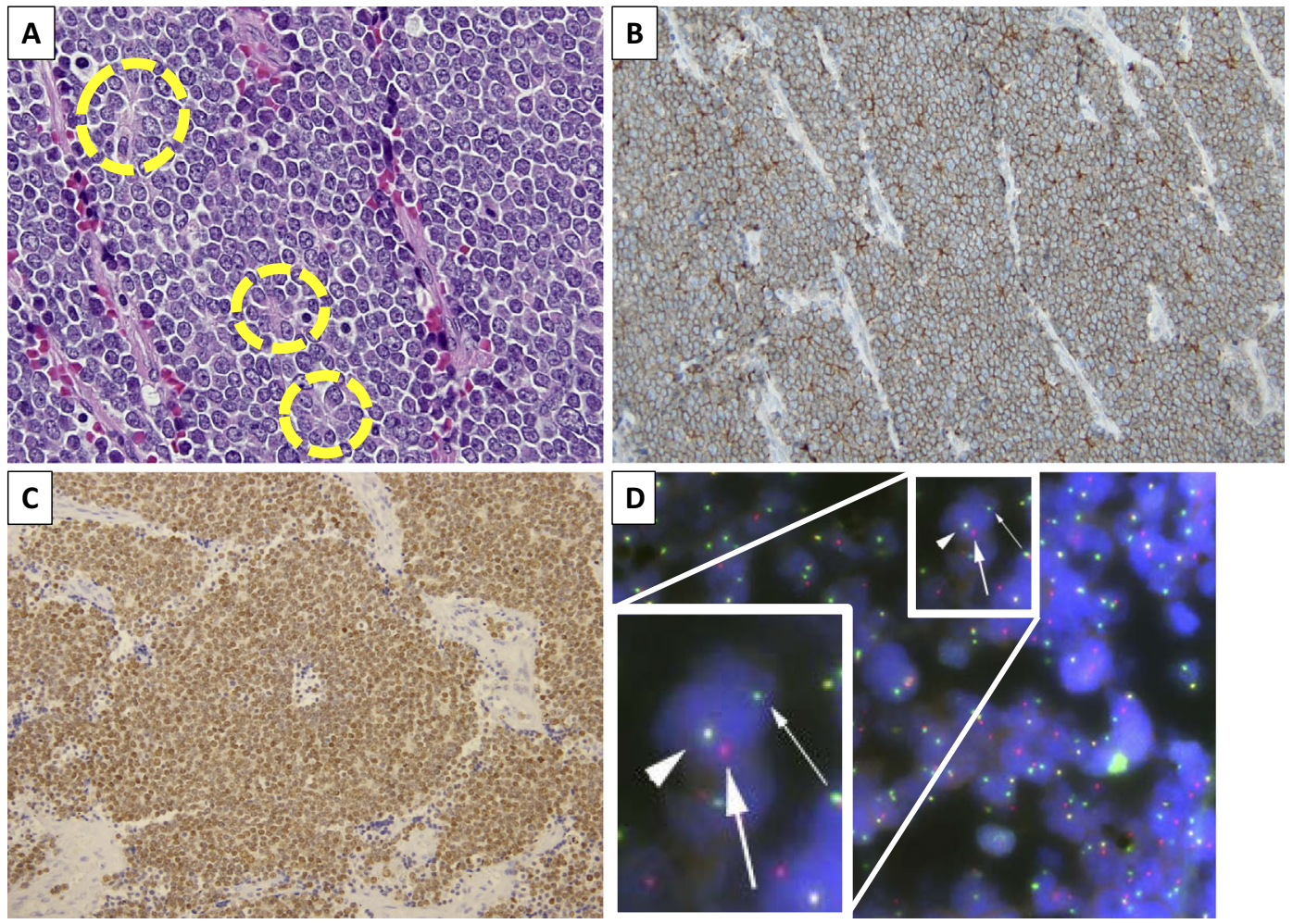

Figure 2. Pathological findings of the resected primary tumor. A: Hematoxylin-Eosin staining shows tumor cells with round nuclei and pale cytoplasm and the formation of rosette structures (yellow circles) $(\times 40$ magnification $)$. B: Immunohistochemistry revealed that the tumor cells were positive for CD99 ( $\times 20$ magnification). C: Immunohistochemistry revealed that the tumor cells were positive for Nkx2.2 ( $\times 20$ magnification). D: Separation of the red signal and green signal (arrows) by fluorescence in situ hybridization reveals Ewing's sarcoma breakpoint region 1 gene rearrangement of the tumor cells, while the yellow signal (arrowhead) shows the normal allele.

We obtained the removed tumor tissue from his former hospital, and the specimen was subjected to pathological studies. The tumor was surrounded by normal adrenal tissue, suggesting that the tumor arose in the adrenal gland. Tumor cells with round nuclei and pale cytoplasm, as well as the formation of rosette structures, led us to suspect Ewing's sarcoma (Fig. 2A). Immunohistochemistry revealed that the tumor cells were positive for CD99 and Nkx2.2, well-known markers for Ewing's sarcoma (Fig. 2B and C, respectively). Hematopoietic markers CD45 and CD117, the epithelial marker keratin, and markers for pheochromocytoma chromogranin A and synaptophysin did not stain these cells (data not shown). Furthermore, fluorescence in situ hybridization (FISH) studies demonstrated rearrangement of the Ewing's sarcoma breakpoint region 1 (EWSRl) gene on chromosome 22 (Fig. 2D). These results confirmed that the tumor was Ewing's sarcoma with adrenal origin.

We were unable to treat the patient with conventional chemotherapy because his performance status rapidly worsened. Therefore, we tried administering pazopanib, a multiple kinase inhibitor for sarcomas, which has been reported to be effective in the treatment of Ewing's sarcoma $(5,6)$, but the patient died on the 37th hospital day. The autopsy studies revealed that the right lobe of the liver had been mostly replaced by tumors (Fig. 3A). These tumors contained CD99- positive cells. The immunohistochemistry signal was much weaker than in the primary tumor (Fig. 3B), although we need to consider the fact that the sample was obtained by autopsy. In addition, the tumor cells were more proliferative. These results indicated that the tumors were metastatic Ewing's sarcomas with poorly differentiated features. No tumors were found in the bones or other organs.

\section{Discussion}

Ewing's sarcoma predominantly arises in the bones of children and adolescents; $80 \%$ of cases are diagnosed before the age of 18 (4). Clinical manifestations include pain, a fever, and bone fracture. Anemia and an elevated level of CRP are frequently observed, which is consistent with the laboratory findings in our patient. Treatment strategies include surgery, radiation, and chemotherapy. One commonly used protocol for chemotherapy consists of vincristine, doxorubicin, cyclophosphamide plus ifosfamide, and etoposide (VDCIE) (7). Recent progress in chemotherapy has greatly improved the survival rate of patients with localized tumors, but the prognosis remains poor in those with metastasis or recurrent tumors $(3,4)$.

Ewing's sarcoma is regarded as a bone tumor in general, but the cell of origin remains elusive. One proposed hy- 

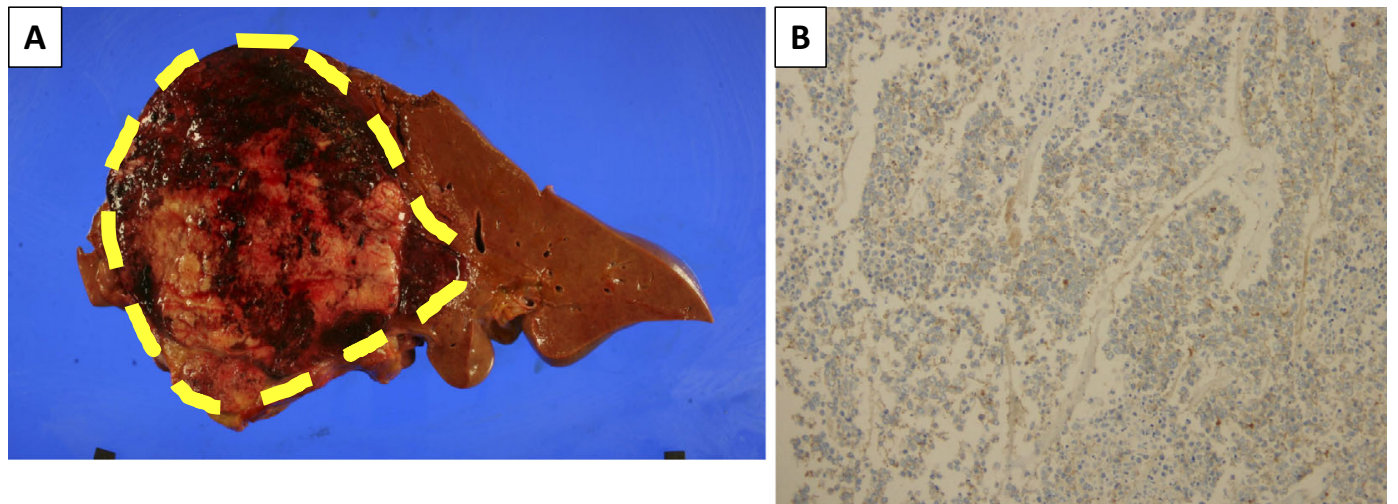

Figure 3. Autopsy studies. A: The right lobe of the liver had been mostly replaced by tumor tissue (yellow circle). B: The liver tumors contained CD99-positive cells ( $\times 20$ magnification).

pothesis is that this type of sarcoma is derived from neuronal cells (8). Elevated levels of neuronal tumor markers, such as NSE and ProGRP, are frequently observed, as seen in our case. Ewing's sarcoma is defined by the chromosomal translocation of EWSR1, which produces a fusion protein composed of Ewing sarcoma protein (EWS) and ETS family transcription factors (9). The fusion protein is thought to regulate the transcription of many genes and to be involved in several oncogenic aspects of the sarcoma cells, including the cell proliferation, survival, and metastasis $(10,11)$. The chromosomal translocation was confirmed by FISH studies in our case, which confirmed the diagnosis of Ewing's sarcoma.

Adenomas account for approximately $80 \%$ of adrenal incidentalomas, regardless of whether or not they are functional. The remaining $20 \%$ include pheochromocytoma (7\%), adrenocortical carcinoma (8\%), and metastasis (5\%) (2). These data indicate that malignant tumors with adrenal origin are very rare, except for adrenocortical carcinoma or malignant pheochromocytoma. In general, MRI studies of Ewing's sarcoma show isointensity to hyperintensity on T1-weighted images and hyperintensity on T2weighted images (12). However, this was not always the case when spinal sarcoma cases were studied (13). Indeed, the findings in our patient were not consistent with typical findings because the tumor was hypointense on T1-weighed images. In addition, large tumors often appear heterogeneous because of internal necrosis and hemorrhaging. Therefore, it is difficult to make a diagnosis of adrenal Ewing's sarcoma based on imaging studies. In contrast, FDG-PET studies are reported to be useful for the staging and restaging of patients with Ewing's sarcoma in terms of sensitivity, specificity, and accuracy (14). The absence of the uptake of the tracer outside the adrenal gland in the first FDG-PET study, as well as the fact that the tumor was surrounded by normal adrenal tissue, indicated that Ewing's sarcoma in our patient arose in the adrenal gland. The autopsy studies also confirmed that there were no tumors in the bones or other organs, except for the liver and the already resected adrenal gland.
Ewing's sarcoma arises at extraskeletal sites in $20-30 \%$ of the patients $(3,15,16)$. The frequent primary sites include the trunk, extremities, head and neck, and retroperitoneum (17). The oldest reported case was 85 years old for skeletal Ewing's sarcoma and 77 years old for extraskeletal sarcoma $(18,19)$. In general, Ewing's sarcoma with extraskeletal origin is more frequent in elderly patients, and both elderly and extraskeletal cases are associated with a poor prognosis $(15,20,21)$. However, some recent reports have shown that the prognoses of these cases were not severe $(22,23)$. It is speculated that recent advances in chemotherapy have gradually improved the prognoses in these cases. Only 13 cases of Ewing's sarcoma with adrenal origin had been reported, according to a literature review published in 2013 (24). The mean age of patients was 22.8 years, and the oldest patient was 57 years old. Recently, additional reports regarding adrenal Ewing's sarcoma have been published (25-31), and the oldest case among those was 63 years old. Therefore, to our knowledge, the present 74-yearold patient is the oldest reported patient with adrenal Ewing's sarcoma. This case suggests that we need to consider Ewing's sarcoma when encountering adrenal tumors, even if the patient is an elderly person. Although the prognosis of adrenal Ewing's sarcoma is poor, the early diagnosis by CD99 staining and analyses for EWSRl gene arrangement can result in a better outcome.

The authors state that they have no Conflict of Interest (COI).

\section{References}

1. Zeiger MA, Siegelman SS, Hamrahian AH. Medical and surgical evaluation and treatment of adrenal incidentalomas. J Clin Endocrinol Metab 96: 2004-2015, 2011.

2. Fassnacht M, Arlt W, Bancos I, et al. Management of adrenal incidentalomas: European Society of Endocrinology Clinical Practice Guideline in collaboration with the European Network for the Study of Adrenal Tumors. Eur J Endocrinol 175: G1-G34, 2016.

3. Balamuth NJ, Womer RB. Ewing's sarcoma. Lancet Oncol 11: 184-192, 2010.

4. Karosas AO. Ewing's sarcoma. Am J Health Syst Pharm 67: 1599- 
$1605,2010$.

5. Alcindor T. Response of refractory Ewing sarcoma to pazopanib. Acta Oncol 54: 1063-1064, 2015.

6. Ranieri G, Mammì M, Donato Di, et al. Pazopanib a tyrosine kinase inhibitor with strong anti-angiogenetic activity: a new treatment for metastatic soft tissue sarcoma. Crit Rev Oncol Hematol 89: 322-329, 2014.

7. Grier HE, Krailo MD, Tarbell NJ, et al. Addition of ifosfamide and etoposide to standard chemotherapy for Ewing's sarcoma and primitive neuroectodermal tumor of bone. $\mathrm{N}$ Engl $\mathrm{J}$ Med 348 : 694-701, 2003

8. Jaffe R, Santamaria M, Yunis EJ, et al. The neuroectodermal tumor of bone. Am J Surg Pathol 8: 885-898, 1984.

9. Delattre O, Zucman J, Plougastel B, et al. Gene fusion with an ETS DNA-binding domain caused by chromosome translocation in human tumours. Nature 359: 162-165, 1992.

10. Lessnick SL, Ladanyi M. Molecular pathogenesis of Ewing sarcoma: new therapeutic and transcriptional targets. Annu Rev Pathol 7: 145-159, 2012.

11. Toomey EC, Schiffman JD, Lessnick SL. Recent advances in the molecular pathogenesis of Ewing's sarcoma. Oncogene 29: 45044516, 2010.

12. Javery $\mathrm{O}$, Krajewski $\mathrm{K}$, O'Regan $\mathrm{K}$, et al. A to $\mathrm{Z}$ of extraskeletal Ewing sarcoma family of tumors in adults: imaging features of primary disease, metastatic patterns, and treatment responses. AJR Am J Roentgenol 197: W1015-W1022, 2011.

13. Tsutsumi S, Yasumoto Y, Manabe A, Ogino I, Arai H, Ito M. Magnetic resonance imaging appearance of primary spinal extradural Ewing's sarcoma: case report and literature review. Clin Neuroradiol 23: 81-85, 2013.

14. Treglia G, Salsano M, Stefanelli A, Mattoli MV, Giordano A, Bonomo L. Diagnostic accuracy of ${ }^{18}$ F-FDG-PET and PET/CT in patients with Ewing sarcoma family tumours: a systematic review and a meta-analysis. Skeletal Radiol 41: 249-256, 2012.

15. Maki RG. Pediatric sarcomas occurring in adults. J Surg Oncol 97: 360-368, 2008

16. Applebaum MA, Worch J, Matthay KK, et al. Clinical features and outcomes in patients with extraskeletal Ewing sarcoma. Cancer 117: 3027-3032, 2011

17. Raney RB, Asmar L, Newton WA, et al. Ewing's sarcoma of soft tissues in childhood: a report from the Intergroup Rhabdomyosarcoma Study, 1972 to 1991. J Clin Oncol 15: 574-582, 1997.

18. Monument MJ, Grossmann AH, Baker CC, Randall RL, Liu T, Albertson DJ. Molecular confirmation of Ewing sarcoma in an 85- year-old woman. Int J Surg Pathol 23: 500-504, 2015.

19. Cheung CC, Kandel RA, Bell RS, Mathews RE, Ghazarian DM. Extraskeletal Ewing sarcoma in a 77-year-old woman. Arch Pathol Lab Med 125: 1358-1360, 2001.

20. Verma V, Denniston KA, Lin CJ, Lin C. A comparison of pediatric vs. adult patients with the Ewing sarcoma family of tumors. Front Oncol 7: 82, 2017.

21. Wolden SL, Alektiar KM. Sarcomas across the age spectrum. Semin Radiat Oncol 20: 45-51, 2010.

22. Valdes M, Nicholas G, Verma S, Asmis T. Systemic therapy outcomes in adult patients with Ewing sarcoma family of tumors. Case Rep Oncol 10: 462-472, 2017.

23. Ahmed SK, Robinson SI, Okuno SH, Rose PS, Laack NN. Adult ewing sarcoma: survival and local control outcomes in 102 patients with localized disease. Sarcoma 2013: 681425, 2013.

24. Abi-Raad R, Manetti GJ, Colberg JW, Hornick JL, Shah JG, Prasad ML. Ewing sarcoma/primitive neuroectodermal tumor arising in the adrenal gland. Pathol Int 63: 283-286, 2013.

25. Blas JV, Smith ML, Wasif N, Cook CB, Schlinkert RT. Ewing sarcoma of the adrenal gland: a rare entity. BMJ Case Rep 2013: 2013.

26. Sasaki T, Onishi T, Yabana T, Hoshina A. Ewing's sarcoma/primitive neuroectodermal tumor arising from the adrenal gland: a case report and literature review. Tumori 99: e104-e106, 2013.

27. Yoon JH, Kim H, Lee JW, et al. Ewing sarcoma/peripheral primitive neuroectodermal tumor in the adrenal gland of an adolescent: a case report and review of the literature. J Pediatr Hematol Oncol 36: e456-e459, 2014.

28. Zahir MN, Ansari TZ, Moatter T, Memon W, Pervez S. Ewing's sarcoma arising from the adrenal gland in a young male: a case report. BMC Res Notes 6: 533, 2013.

29. Tsang YP, Lang BH, Tam SC, Wong KP. Primitive neuroectodermal adrenal gland tumour. Hong Kong Med J 20: 444-446, 2014.

30. Zhang L, Yao M, Hisaoka M, Sasano H, Gao H. Primary Ewing sarcoma/primitive neuroectodermal tumor in the adrenal gland. APMIS 124: 624-629, 2016.

31. Pal DK, Chandra V, Ranjan KR, Chakrabortty D, Banerjee M. Ewing's sarcoma of the adrenal gland. APSP J Case Rep 7: 20, 2016.

The Internal Medicine is an Open Access article distributed under the Creative Commons Attribution-NonCommercial-NoDerivatives 4.0 International License. To view the details of this license, please visit (https://creativecommons.org/licenses/ by-nc-nd/4.0/).

(C) 2018 The Japanese Society of Internal Medicine Intern Med 57: 551-555, 2018 